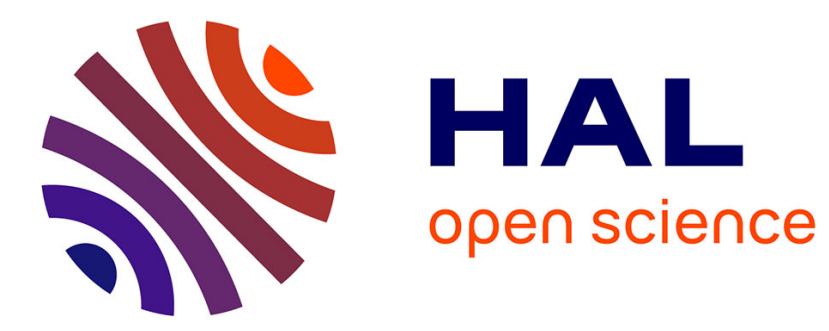

\title{
Stress analysis around crack tips in finite strain problems using the eXtended finite element method
}

Grégory Legrain, Nicolas Moes, Erwan Verron

\section{To cite this version:}

Grégory Legrain, Nicolas Moes, Erwan Verron. Stress analysis around crack tips in finite strain problems using the eXtended finite element method. International Journal for Numerical Methods in Engineering, 2005, 63 (2), pp.290 - 314. 10.1002/nme.1291 . hal-00496953

\section{HAL Id: hal-00496953 https://hal.science/hal-00496953}

Submitted on 2 Jul 2010

HAL is a multi-disciplinary open access archive for the deposit and dissemination of scientific research documents, whether they are published or not. The documents may come from teaching and research institutions in France or abroad, or from public or private research centers.
L'archive ouverte pluridisciplinaire HAL, est destinée au dépôt et à la diffusion de documents scientifiques de niveau recherche, publiés ou non, émanant des établissements d'enseignement et de recherche français ou étrangers, des laboratoires publics ou privés. 


\title{
Stress analysis around crack tips in finite strain problems using the eXtended Finite Element Method
}

\author{
G. Legrain ${ }^{1}-$ N. Moës ${ }^{1}$ - E. Verron ${ }^{1}$ \\ ${ }^{1}$ GeM - Institut de Recherche en Génie Civil et Mécanique \\ Ecole Centrale de Nantes - Université de Nantes - CNRS UMR 6183 \\ 1, rue de la Noë, BP 92101 - 44321 Nantes Cedex 3 - FRANCE
}

\begin{abstract}
Fracture of rubber-like materials is still an open problem. Indeed, it deals with modeling issues (crack growth law, bulk behaviour) and computational issues (robust crack growth in $2 \mathrm{D}$ and $3 \mathrm{D}$, incompressibility). The present study focuses on the application of the eXtended Finite Element Method (X-FEM) to large strain fracture mechanics for plane stress problems. Two important issues are investigated: the choice of the formulation used to solve the problem and the determination of suitable enrichment functions. It is demonstrated that the results obtained with the method are in good agreement with previously published works.
\end{abstract}

\section{INTRODUCTION}

As pointed out in [1], fracture of rubber-like materials is still an open problem. Indeed, very few tools exist to solve this particular kind of problems. They cannot avoid the remeshing issue coming from crack growth, which for some of them must be handled by the user at each step of the propagation of the crack. These problems contrast with linear fracture mechanics, where simulation tools have improved over the past years. Meshless methods [2] has been developed to avoid remeshing by enriching the approximation basis with discontinuous modes. Finite element approaches have also been modified within the partition of unity framework [3]. One of these methods is the eXtended Finite Element Method (referred as X-FEM throughout the rest of the paper). This method was first proposed as a response to the remeshing issue in crack propagation in the context of linear fracture mechanics $[4,5]$. The X-FEM uses the Partition of Unity in two ways: first to take into account the displacement jump across the crack faces far away from the crack tip, and second to enrich the approximation close to the tip considering the asymptotic 
fields. This method exhibits advantages which are in common with meshless methods (enrichment of the interpolation basis) and with the finite element method (mesh based approximations). Several fracture mechanics applications have been solved with the X-FEM approach: crack growth with friction [6], arbitrary branched and intersecting cracks [7], three dimensional crack propagation $[8,9]$ and cohesive crack simulation $[10,11]$. The method has also been coupled with the level set approach [12, 13] for greater robustness and versatility. The main purpose of the present paper is to show how to solve nonlinear fracture mechanics problems with the X-FEM, particularly for rubberlike materials. It seems that only one paper has been published on this problem: this paper, presented by Dolbow and Devan [14] concerns geometrically nonlinear fracture with the X-FEM. More precisely, it investigates the locking issue occurring in plane strain analysis. Here, emphasis is placed on the enrichment around the crack tip in nonlinear fracture mechanics for plane stress case. The robustness and versatility of the X-FEM in nonlinear fracture mechanics is demonstrated. This paper is organized as follows: in the next section, the governing equations of the problem are discussed. Then, technical aspects of the implementation of the eXtended Finite Element Method to nonlinear elasticity are presented, followed by numerical examples of this implementation which show the versatility of the method. Finally, we will conclude and present possible extensions to this work.

\section{GOVERNING EQUATIONS}

\subsection{Deformation of a cracked body in large strain}

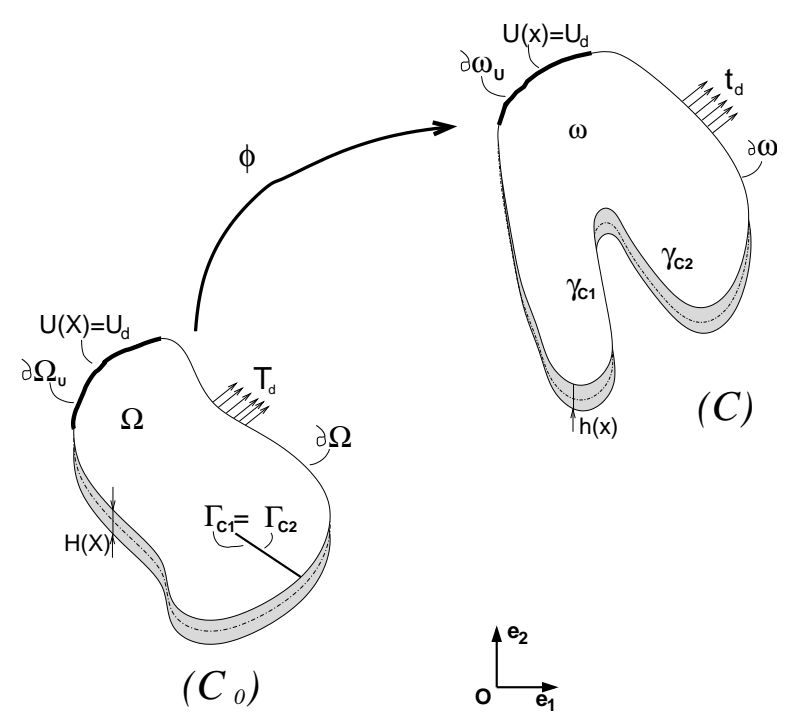

Figure 1: Notations of the model problem.

Consider a thin cracked sheet $\mathcal{B}$ defined by its mid-plane surface $\Omega$ and its thickness 
distribution $H$ in the reference undeformed configuration $\left(\mathcal{C}_{0}\right)$. Under plane mechanical loading, $\mathcal{B}$ deforms and occupies the configuration $(\mathcal{C})$. The corresponding mid-plane surface, boundary and thickness distribution are respectively denoted $\omega, \partial \omega$ and $h$. Figure 1 presents the notations. The motion between $\left(\mathcal{C}_{0}\right)$ and $(\mathcal{C})$ can be described by the mapping $\phi$ which relates the current position of a particle, $\mathbf{x}$, at time $t$, to its initial position $\mathbf{X}$ :

$$
\mathbf{x}=\phi(\mathbf{X}, t)
$$

In the deformed configuration, the boundary of $\mathcal{B}$ can be split into two disjoint sets: $\partial \omega_{u}$ on which the displacement field is enforced (Dirichlet boundary condition), and $\partial \omega_{T}$ on which the surface traction is enforced (Neumann boundary condition). More precisely, $\partial \omega_{T}$ includes the crack faces $\gamma_{C 1}$ and $\gamma_{C 2}$. The corresponding parts of the boundaries in the reference configuration are denoted $\partial \Omega_{u}$ (Dirichlet boundary condition), and $\partial \Omega_{T}$ (Neumann boundary condition), and $\Gamma_{C 1}$ and $\Gamma_{C 2}$ for both crack faces. Mathematically, these crack boundaries are different because of the bijectivity of the mapping $\phi$, even if they are superimposed in $\left(\mathcal{C}_{0}\right)$, as shown in Fig. 1. Considering that there is no body forces, the strong form of the boundary value problem in the material description is :

$$
\begin{array}{ll}
\operatorname{Div}_{\mathbf{X}} \underline{\underline{P}}=\mathbf{0} & \text { in } \Omega \\
\mathbf{u}(\mathbf{x})=\mathbf{u}_{\mathbf{d}} & \text { on } \partial \Omega_{u} \\
\underline{\underline{P}} \cdot \mathbf{N}=\mathbf{T}_{\mathbf{d}} & \text { on } \partial \Omega_{T} \backslash\left(\left(\Gamma_{C 1}\right) \cup\left(\Gamma_{C 2}\right)\right) \\
\underline{\underline{P}} \cdot \mathbf{N}=\mathbf{0} & \text { on } \Gamma_{C 1} \text { and } \Gamma_{C 2}
\end{array}
$$

In these equations $\underline{\underline{P}}$ is the first Piola-Kirchhoff stress tensor, $\mathbf{u}$ is the displacement field, $\mathbf{u}_{\mathbf{d}}$ is the prescribed displacement field, $\mathbf{N}$ stands for the unit outward normal vector to the boundary $\partial \Omega_{T}$ and $\mathbf{T}_{\mathbf{d}}$ is the prescribed Piola-Kirchhoff traction vector, i.e. the force measured per unit of reference boundary length. According to these equations, the principle of virtual work in the material description is expressed as :

$$
\mathcal{F}(\mathbf{u}, \delta \mathbf{u})=\int_{\Omega} H(X) \underline{\underline{S}}: \delta \underline{\underline{E}} d \Omega-\int_{\partial \Gamma_{T}} \mathbf{T}_{\mathbf{d}} \cdot \delta \mathbf{u} d \Gamma=0 \quad \forall \delta \mathbf{u}
$$

in which $\underline{\underline{S}}$ is the second Piola-Kirchhoff stress tensor, $\underline{\underline{E}}$ the Green-Lagrange strain tensor and $\delta \overline{\mathbf{u}}$ represents the virtual displacement field. In the case of rubber-like materials, the above equation is highly nonlinear (due to both large strain and material nonlinearities) and its linearization is an essential prerequisite for the use of a Newton-like algorithm :

$$
\begin{aligned}
& D \mathcal{F}(\mathbf{u}, \delta \mathbf{u})[\mathbf{u}]=\int_{\Omega} H(X) D \underline{\underline{E}}[\delta \mathbf{u}]: \underline{\underline{\underline{\mathcal{C}}}}: D \underline{\underline{E}}[\mathbf{u}] d \Omega \\
& \quad+\int_{\Omega} H(X) \underline{\underline{S}}:\left[\left(\operatorname{Grad}_{X} \mathbf{u}\right)^{T} \cdot\left(\operatorname{Grad}_{X} \delta \mathbf{u}\right)\right] d \Omega
\end{aligned}
$$

where $D \bullet$ is the directional derivative operator and $\underline{\underline{\underline{\mathcal{C}}}}$ is the material elasticity tensor. Here, the prescribed tractions are assumed not to $\overline{\overline{\text { depend }}}$ on the displacement field. Finally, the constitutive equation of the material is examined. The material is supposed 
homogeneous, isotropic and incompressible. Moreover the general theory of hyperelasticity is considered. Here the material is assumed to obey the Neo-Hookean constitutive equation. The corresponding strain energy function is :

$$
W=\frac{\mu}{2}(\operatorname{Tr} \underline{\underline{C}}-3)
$$

where $\mu$ is the shear modulus and $\underline{\underline{C}}$ stands for the right Cauchy-Green dilatation tensor $(\underline{\underline{C}}=\underline{\underline{I}}+2 \underline{\underline{E}})$. The second Piola-Kirchhoff stress tensor is given by :

$$
\underline{\underline{S}}=2 \frac{\partial W}{\partial C}-p \underline{\underline{C}}^{-1}=\mu \underline{\underline{I}}-p \underline{\underline{C}}^{-1}
$$

In this equation $p$ is the hydrostatic pressure due to the incompressibility of the material, it is determined using the equilibrium equations. Because of the small thickness of the sheet and plane loading, the plane stress assumption is adopted. In this case, the thickness direction is irrelevant and the thickness variation is simply given by :

$$
h^{2}=\frac{H^{2}}{\operatorname{det}(\underline{\underline{\bar{C}}})}
$$

where $h$ is the thickness distribution in the deformed configuration and $\underline{\underline{C}}$ is the in-plane dilatation tensor. As a consequence, the hydrostatic pressure can explicitly be evaluated and the in-plane second Piola-Kirchhoff stress tensor is :

$$
\underline{\underline{\underline{S}}}=\mu\left[\underline{\underline{\bar{I}}}-\operatorname{det}(\underline{\underline{\bar{C}}})^{-1} \underline{\underline{C}}^{-1}\right]
$$

where $\underline{\underline{I}}$ is the $2 \times 2$ identity tensor. Finally the $4^{\text {th }}$ order elasticity tensor $\underline{\underline{\underline{\mathcal{C}}}}$ of Eq. (4) reduces to :

$$
\underline{\underline{\underline{\underline{C}}}}=2 \mu \operatorname{det}(\bar{C})^{-1}\left(\underline{\underline{\bar{C}}}^{-1} \otimes \underline{\underline{C}}^{-1}+\underline{\underline{\underline{I}}}\right)
$$

where $\underline{\underline{\underline{I}}}=\partial \underline{\underline{C}}^{-1} / \partial \underline{\underline{C}}^{-1}$.

\subsection{Fracture of rubber-like materials}

Rivlin and Thomas proposed an extension of the energy release rate initially proposed by Griffith [15] to rubber-like materials [16]. In their work, they showed that the fracture of rubbers is controlled by the tearing energy $T$ defined by :

$$
T=-\left(\frac{\partial W}{\partial A}\right)_{l}
$$

where $W$ stands for the strain energy, $A$ the area of one surface of the crack, and the subscript $l$ indicates that the differentiation is carried out for constant displacement on 
the parts of the boundary which are not traction free. Considering that $A=c H$ (where $H$ stands for the thickness of the plate and $c$ the crack length), Eq. (10) becomes:

$$
T=-\left(\frac{1}{H} \frac{\partial W}{\partial c}\right)
$$

Numerically, the computation of this tearing energy will be performed using the Rice integral $J$ which is commonly used in linear fracture mechanics to quantify the singularity of the stress field near the crack tip :

$$
J=\int_{\gamma}\left(w n_{1}-\mathbf{n} \cdot \underline{\underline{\sigma}} \cdot \frac{\partial \mathbf{u}}{\partial x_{1}}\right) d \gamma
$$

in which, $\gamma$ is the integration contour, $\mathbf{n}$ is the unit normal outward vector, and $w$ the

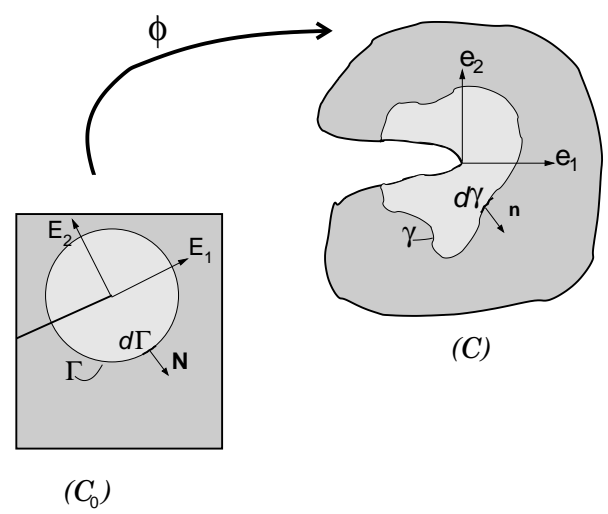

Figure 2: Contour used for the $J$-integral.

strain energy function per unit of deformed volume as shown in Figure 2. For large strain problems, it is possible to express the $J$-integral in the material description. It can be established by the use of a pull-back operation on variables involved in Eq. (12) and described in Fig. 2 :

$$
J=\int_{\Gamma}\left(W N_{1}-\mathbf{N} \cdot \underline{\underline{P}} \cdot \frac{\partial \mathbf{u}}{\partial X_{1}}\right) d S
$$

In order to demonstrate this result, consider the material expression of the Eshelby stress tensor for hyperelastic materials [17] :

$$
\underline{\underline{M}}=W \underline{\underline{I}}-\underline{\underline{F}}^{T} \cdot \underline{\underline{P}}
$$

Then, in the case of unloaded crack surfaces and crack tip, the following expression is equivalent to Eq. (13) :

$$
J=\int_{\Gamma} \mathbf{E}_{1} \cdot \underline{\underline{\tilde{M}}} \cdot \mathbf{N} d \Gamma
$$


with :

$$
\underline{\underline{\underline{M}}}=\underline{\underline{M}}-\underline{\underline{P}}
$$

where $\mathbf{N}$ is the outward normal vector to $\Gamma$, and $E_{1}$ the tangential vector to the crack. This expression of the $J$-integral is not well-suited to the finite element context because integration over a contour is difficult in FEA: the interpolation of material variables on the contour leads to numerical errors whereas those material variables are well-known at the quadrature points inside the elements. In fact, expressing $\mathrm{J}$ in domain form is better suited to finite element computation. Following the procedure proposed in [18, 19], it is possible to express $J$ in domain form :

$$
J=\left[-\int_{S} \underline{\underline{\tilde{M}}} \cdot \operatorname{Grad}_{X}(\alpha) d \Omega\right] \cdot \mathbf{E}_{1}
$$

where $S$ is the area bounded by $\Gamma$, and $\alpha$ represents a weight function that is sufficiently smooth on $S$, vanishes on $\Gamma$, and is equal to 1 at the crack-tip. This domain expression of the $J$-integral is equivalent to the contour integral Eq. (15), and may be rewritten as

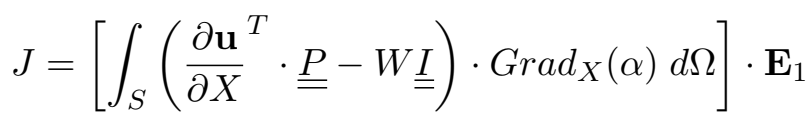

\section{APPLICATION OF THE X-FEM TO LARGE STRAIN FRACTURE MECHANICS}

In this section, we describe how the X-FEM can be adapted to nonlinear fracture mechanics. The X-FEM concept has already been applied in the finite strain context in [14] where the locking issue in plane strain was discussed. However, the authors did not provide detailed implementation aspects of the X-FEM, focusing on the implementation of the enhanced assumed strain method. The present section focuses on the implementation issue in a more detailed manner. First, generalities on the X-FEM are reviewed. Then, an appropriate enrichment strategy for nonlinear fracture mechanics is proposed, and finally the choice of the total lagrangian formulation is justified in regard to the numerical procedure.

\subsection{X-FEM discretization}

The formulation used in this study is the total lagrangian formulation i.e. the displacement field is approximated on the initial configuration. With classical finite elements, the approximation of a vector field $\mathbf{u}(\mathbf{X})$ on an element $\Omega_{e}$ is written as:

$$
\left.\mathbf{u}(\mathbf{X})\right|_{\Omega_{e}}=\sum_{\alpha=1}^{n} u^{\alpha} \mathbf{N}^{\alpha}(\mathbf{X})
$$


where $n$ is the number of coefficients describing the approximation over the element, $u^{\alpha}$ is the $\alpha^{\text {st }}$ nodal value of this approximation and $\mathbf{N}^{\alpha}$ is the classical vectorial shape function associated with dof $\alpha$. Within the partition of unity, the approximation is enriched as:

$$
\left.\mathbf{u}(\mathbf{X})\right|_{\Omega_{e}}=\sum_{\alpha=1}^{n} \mathbf{N}^{\alpha}\left(u^{\alpha}+\sum_{\beta=1}^{n_{e}} a_{\beta}^{\alpha} \phi_{\beta}(\mathbf{X})\right)
$$

where $n_{e}$ is the number of enrichment modes, $a_{\beta}^{\alpha}$ is the additional dof associated to dof $\alpha$ and $\phi_{\beta}$ stands for the $\beta^{\text {th }}$ scalar enrichment function. Two different types of enrichment are considered in fracture mechanics (see [5]), they are presented in Figure 3: A discontinuous enrichment for nodes whose support is bisected by the crack. In this case, the interpolation of the displacement field is discontinuous across the crack. A Heaviside jump function is considered to model the discontinuity: this function is equal to +1 on one side of the crack and -1 on the other side. The associated dof manages the magnitude of this displacement discontinuity, as seen in Fig. 3(a). The Heaviside function is computed using the level set representation of the crack $[20,8]$.

The second type of enrichment is the near tip enrichment for nodes whose support contains the crack tip. In linear elastic fracture mechanics, the enrichment functions are determined using the asymptotic displacement field near the tip of the crack (see Fig. 3(b)). The problem in nonlinear elasticity is the determination of this asymptotic field. This will be discussed in the next section.

Finally, we note that the modified Gauss quadrature scheme described in [5] is used to integrate both discontinuous and non-polynomial functions over the elements.

\subsection{Near tip enrichment}

As pointed out in various works [21, 22], the choice of the right enrichment function is fundamental in the X-FEM in order to achieve precision even with coarse meshes. Usually, enrichment functions are related to the asymptotic displacement field around the crack tip. The determination of this field is a complex topic since the problem is highly nonlinear. Theoretical results were established in the incompressible plane stress case by Geubelle and Knauss[23, 24, 25], in the general plane strain case by Knowles and Sternberg [26, 27] and in the incompressible plane strain case by Stephenson [28]. These studies were conducted using the generalized Neo-Hookean strain energy: recall that in the present case, the incompressible plane stress assumption is adopted and the classical Neo-Hookean material is considered. Thus, the corresponding asymptotic field is given by [23]:

$$
\left\{\begin{array}{l}
v_{1}(r, \theta) \approx r \cos (\theta) \\
v_{2}(r, \theta)=r^{1 / 2} \sin (\theta / 2)
\end{array}\right.
$$

where the $v_{1}$ and $v_{2}$ are the displacement vector projected respectively on $\mathbf{E}_{1}$ and $\mathbf{E}_{2}$, and $(r, \theta)$ describes the crack front coodinate system (see Fig. 2). Moreover, if second order terms are needed, it can be shown that only one additional term appears along 


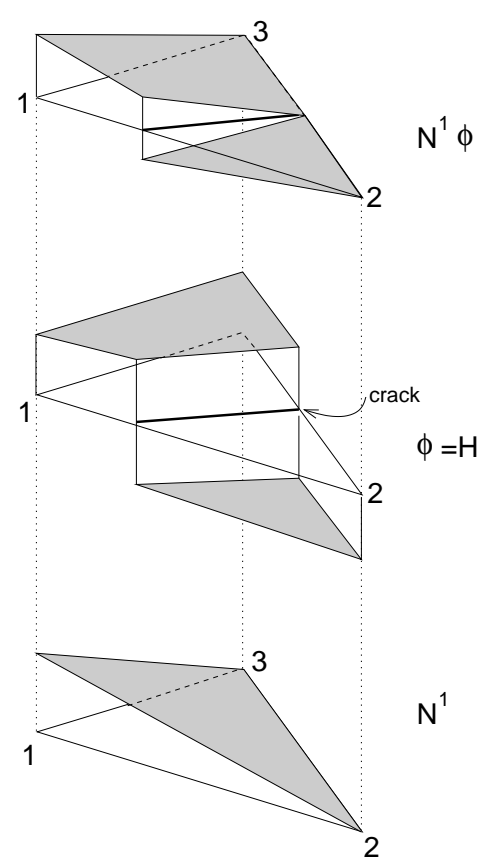

(a)

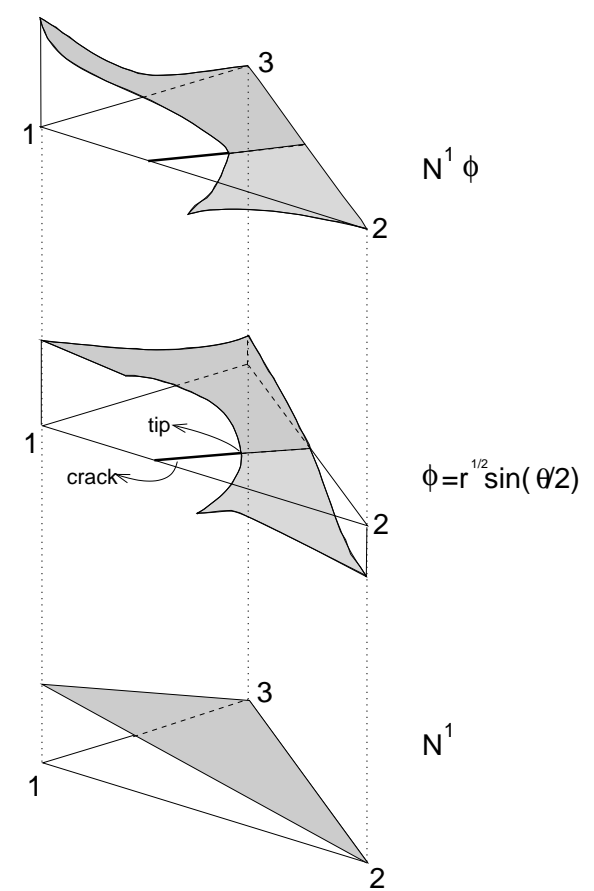

(b)

Figure 3: (a) Discontinuous approximation function obtained by the product of the regular function $\left(N^{1}\right)$ and the enrichment function $(\phi)$, (b) Near tip approximation function obtained by the product of the regular function $\left(N^{1}\right)$ and the enrichment function $(\phi)$.

the second axis. However, this term is defined by an implicit equation, thus it is not straightforward to use it numerically as enrichment. Finally the enrichment function that will be used in the following is:

$$
\phi=\left\{r^{1 / 2} \sin (\theta / 2)\right\}
$$

As a comparison, in linear fracture mechanics, the enrichment functions are:

$$
\phi=\left\{r^{1 / 2} \sin (\theta / 2), r^{1 / 2} \cos (\theta / 2), r^{1 / 2} \sin (\theta / 2) \sin (\theta), r^{1 / 2} \cos (\theta / 2) \sin (\theta)\right\}
$$

Indeed, it should be noted that the term on $\mathbf{E}_{1}$ of Eq. (21) reduces to ' $x^{\prime}$ when expressed in the crack front coordinate system. Since this function is linear, it is already contained in the polynomial approximation space. If this term was used in the enrichment basis, it would lead to an ill-conditioned system. Consquently, it is not considered in the following and won't be used as an enrichment function. Note also that (22) is similar to the discontinuous term of Eq. (23).

Remark on the stress singularity around the crack tip:

Using the asymptotic displacement field near the crack tip (22), the asymptotic stress 
and strain fields are:

$$
\begin{array}{r}
\underline{\underline{\sigma}} \longrightarrow \frac{1}{r} \text { as } r \rightarrow 0 \\
\underline{\underline{P}} \longrightarrow \frac{1}{\sqrt{r}} \\
\sqrt{\operatorname{det}(\underline{\underline{\underline{C}}})} \longrightarrow \frac{1}{\sqrt{r}}
\end{array}
$$

As shown in Eqs. (24) and (25), the order of the stress singularity differs between the reference and the current configurations: it is higher in the current configuration. This stems from the fact that the energy release rate, when evaluated on the actual state, must remain finite although the current thickness tends to zero at crack tip $(h=H / \sqrt{\operatorname{det}(\underline{\underline{\underline{C}}})})$. Thus, the change of the singularity order of the stress field balances the thickness evolution.

\subsection{Solution procedure}

As presented above, the total lagrangian formulation of governing equations is considered here. Before detailing the solution procedure itself, the choice of this formulation should be justified. Classically, the integration of the weak form is performed on parent elements. The determination of integrals on a given configuration necessitates the use of the mapping which relates the real element to its parent counterpart. For an ordinary finite element, this mapping is polynomial and well-known for every configuration, particularly the reference and the current configurations. In the context of the X-FEM, additional difficulties exist as shown in Figure 4: due to the enrichment functions, the mapping between the deformed element and its parent counterpart is non polynomial. Nevertheless the mapping between the undeformed element and its parent counterpart remains polynomial. Thus the integration of the weak form is easier over the reference configuration, i.e. using a total lagrangian formulation. The integration configuration now being chosen, the use of the interpolated displacement field in Eq. (3) leads to the discretized system to be solved; and its use in Eq. (4) gives the tangent stiffness matrix.

\section{NUMERICAL EXAMPLES}

In this section, some basic examples are first presented to validate the use of the XFEM for large strain elastic problems. Then nonlinear fracture mechanics examples are considered to exhibit the importance of enrichment. Finally the efficiency of the present method is highlighted.

\subsection{Preliminary validations}

The aim of the following four examples is the validation of the method. The two first examples are devoted to nonlinear structural problems, whereas the last two deal with 


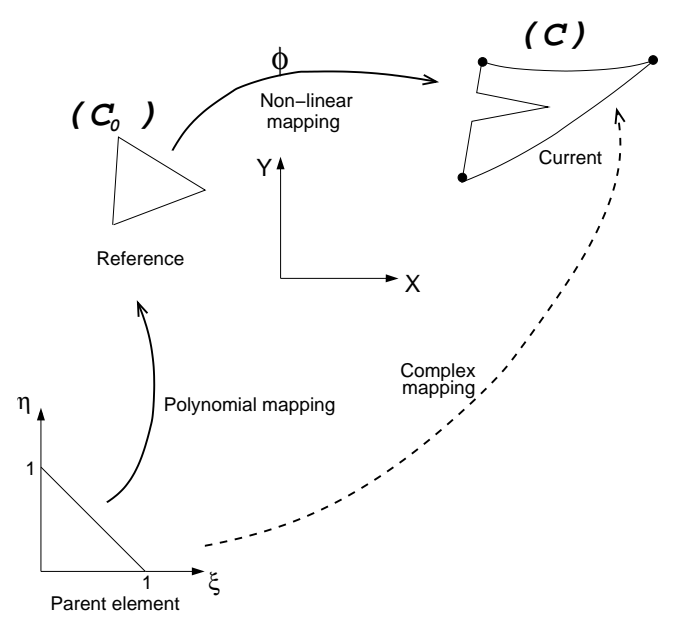

Figure 4: Mappings associated to the eXtended Finite Element Method for an enriched element with large deformations.

nonlinear fracture mechanics.

\subsubsection{Mesh independence}

Consider the uniaxial extension of a single edge notched specimen under tensile loading (SET) as presented in Figure 5(a). Its height and width are both equal to $2 \mathrm{~mm}$. The material is assumed to be Neo-Hookean with $\mu=0.4225 \mathrm{MPa}$, which corresponds to an unfilled natural rubber. The bottom edge is fixed vertically and an additional node is blocked horizontally to prevent rigid body motions. Two different meshes are considered: the first one is a structured $32 \times 16$ triangular elements mesh shown in Figure 5(b), and the second one is a structured $32 \times 15$ triangular elements mesh (see Figure 5(c)). For the first mesh $(32 \times 16)$ the crack is aligned with element edges and for the second mesh $(32 \times 15)$ the crack crosses finite elements. Vertical displacement of the nodes situated on the top edge are compared for the two meshes and the corresponding discrepancy versus the initial horizontal position of these top edge nodes is plotted in Figure 6(a). The difference between the two displacements is smaller than $0.18 \%$ even for large strain as illustrated by the deformed mesh in Figure 6(b). This example demonstrates one of the greatest advantages of the X-FEM: the part can be discretized without taking into account the crack position during mesh generation.

\subsubsection{Comparison with a standard finite element code}

In this example, X-FEM and Abaqus [29] results are compared. A SET specimen (dimensions $=2 \mathrm{~mm} \times 6 \mathrm{~mm}$ and crack length $=1 \mathrm{~mm}$ ) is subjected to a prescribed displacement on its top edge equal to $4 \mathrm{~mm}$ and its bottom edge is fixed. The material is identical to the one considered in section 4.1.1. The mesh used in Abaqus conforms to the crack sur- 


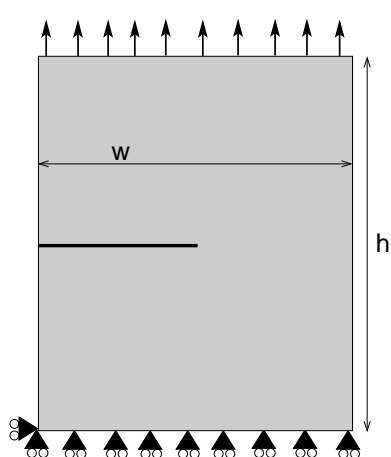

(a)

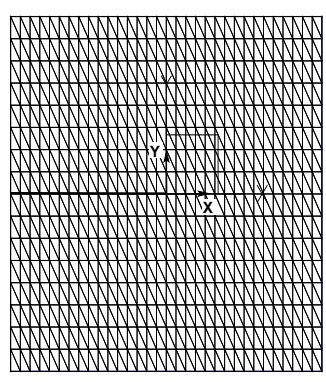

(b)

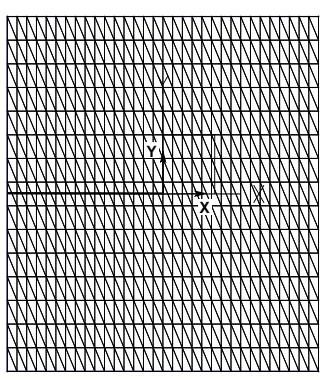

(c)

Figure 5: (a) Single edge notched specimen under uniaxial tensile loading, (b) $32 \times 16$ mesh, (c) $32 \times 15$ mesh.

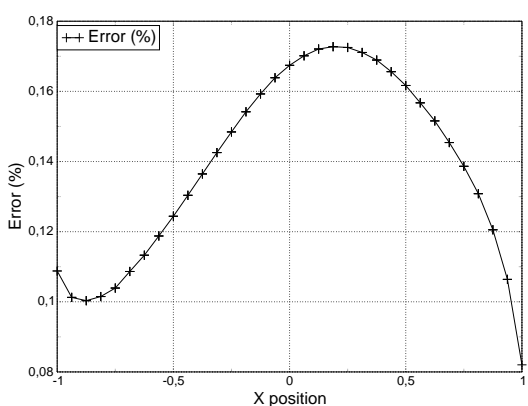

(a)

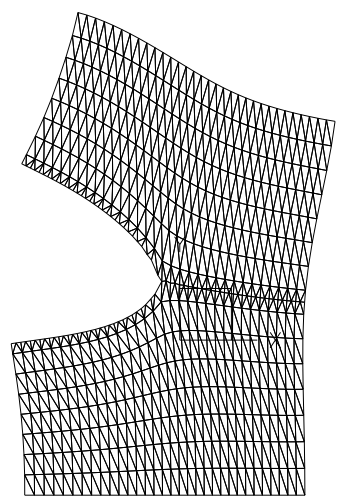

(b)

Figure 6: (a) Error on the displacement of the top edge nodes between the two meshes results, (b) Deformed shape of the SET.

face and has the same element density as the X-FEM mesh which is unstructured (the crack runs through elements). Deformed configurations are compared. Qualitatively, they are similar as shown in Figure 7(a) and 7(b). To more precisely compare them, the vertical displacement of the crack surface nodes versus their initial horizontal position are plotted in Figure 7(c). The deformed shape of the crack is very similar for the two simulations, and results obtained with X-FEM are reliable.

\subsubsection{Domain independence of the $J$-integral}

The aim of this example is to ensure that the computation of the tearing energy does not depend on the domain chosen to calculate the $J$-integral. The problem considered here is identical to the previous one. The $J$-integral is computed for different stretch 


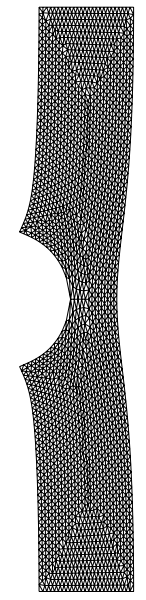

(a)

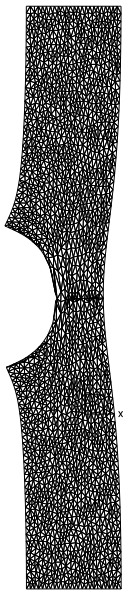

(b)

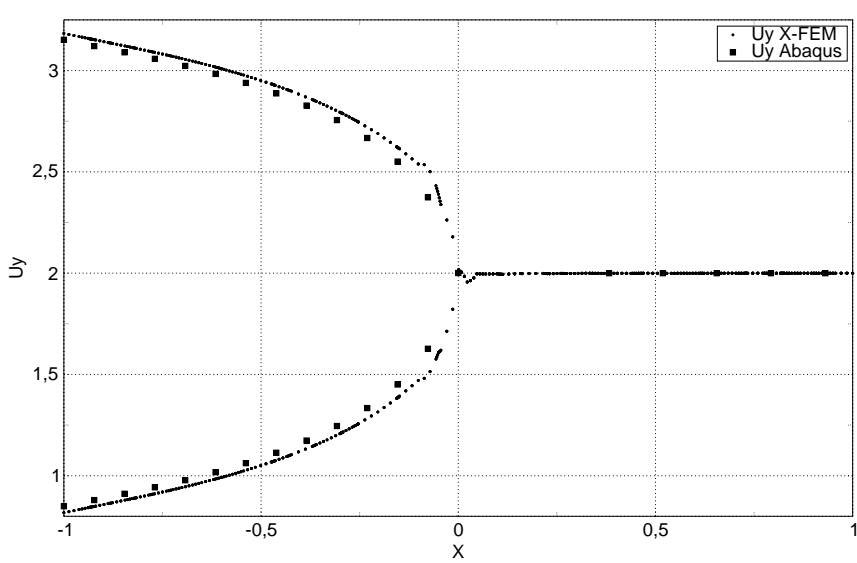

(c)

Figure 7: (a) Abaqus deformed configuration; (b) X-FEM deformed configuration; (c) Comparison of the two crack vertical displacements.

levels and considering different circular integration domains. More precisely, an element is selected in the integration domain if one of its nodes is contained in the circular region. Figure 8 presents the evolution of the $J$-integral (normalized with respect to its converged value) as a function of the integration domain radius (normalized with respect to the characteristic length of the element that contains the crack tip) for various stretch levels. In all cases, the $J$-integral computation converges rapidly (at about 2 layers of elements) and the convergence rate is not significantly influenced by the stretch level.

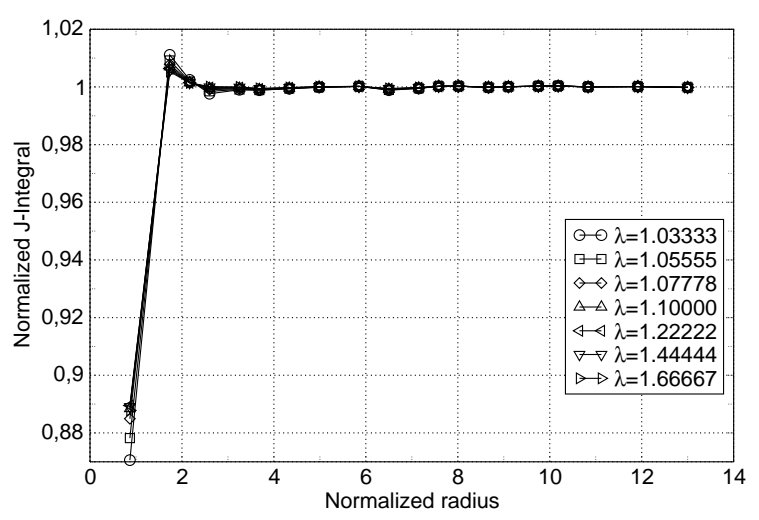

Figure 8: Evolution of the J-integral with respect to both domain size and stretch level $(\lambda)$ 


\subsubsection{Robustness of the enrichment process}

As a last example, we examine the accuracy of the tearing energy computation with respect to the set of enriched nodes. As mentioned in Section 3.1, the number of enriched nodes in fracture problems is small. A number of studies have demonstrated that an accurate computation of the tearing energy is ensured by a sufficiently refined mesh. Nevertheless, for nonlinear fracture problems, computing time highly increases with the number of dofs because of the iterative solving method, and then the tearing energy computation has to be accurate even with coarse meshes in order to be able to investigate complex parts.

Consider a SET specimen $(7 \mathrm{~mm} \times 6 \mathrm{~mm})$ discretized by 84 triangular $1 \mathrm{~mm} \times 1 \mathrm{~mm}$ elements as shown in Figure 9(a). The crack tip position varies from $(-1 ; 0)$ to $(+1 ; 0)$ and the crack emerges from the left edge of the membrane. The set of enriched nodes is represented by gray squares in Figure 9. The bottom edge is fixed, and the top face is subjected to a vertical displacement leading to a stretch of $157 \%$. The radius of the domain used to compute the J-integral is $0.9 \mathrm{~mm}$. The evolution of the tearing energy is plotted with respect to the tip position in Figure 10. It corresponds to the curve denoted "J classical" in Fig. 10. In the neighborhood of $X= \pm 0.5, J$ varies significantly; it corresponds to the change in the set of enriched nodes set when the crack tip moves from an element to another. In order to smooth the evolution of $J$ with the set of enriched nodes, additionally enriched nodes are incorporated to the set by considering a virtual circle around the crack tip (see Figures 9(b) and (c) where gray square represent enriched nodes). Two different circle sizes are studied, their radii are respectively two and three times the characteristic length of the element containing the crack tip. The corresponding result curves are presented in Figure 10. In both cases, the curves are sufficiently smooth to ensure the accuracy of the $J$-integral computation. Moreover, the set of 2 layers is enough for the present problem, as the corresponding results agree well with the fourth curve in Fig. 10 where a twice refined mesh was considered. Consequently, for a smooth variation of the evaluated J-integral as the crack grows, several layers of enrichment should be considered for coarse meshes. We remark that in the following Sections, only one layer of elements will be enriched, because meshes will be considered to be sufficiently refined.

\subsection{Simple loading problems}

In this section, classical problems are considered to study the influence of both enrichment and mesh density. For each example in this section, the evolution of the tearing energy with respect to the stretch level, the mesh size and the enrichment type is examined.

Nonlinear fracture mechanics study for rubber-like material really started in the 1950's with the work of Rivlin and Thomas [16], who showed that the fracture of rubber is controlled by one parameter: the tearing energy. This parameter is equivalent to the energy release rate, and thus to the J-Integral proposed later by Rice [30]. They also introduced a parameter $(k)$ which relates the energy loss due to the crack and the strain 


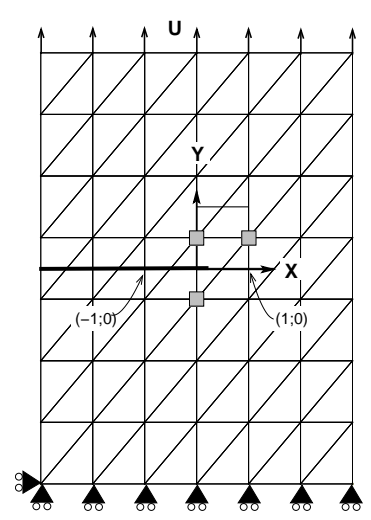

Classical

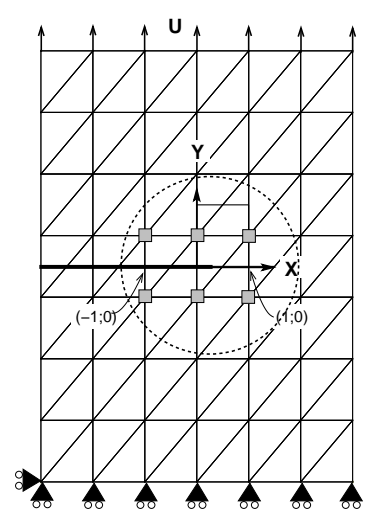

2 layers

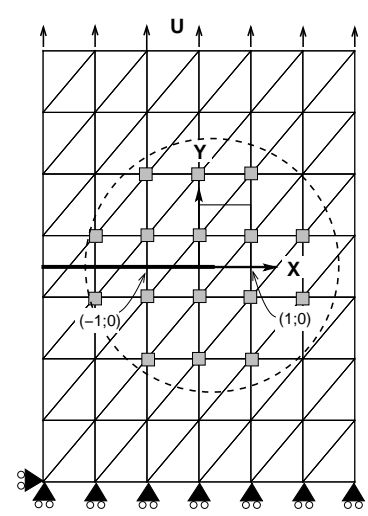

3 layers

Figure 9: The mesh used and the near-tip enriched nodes (gray squares).

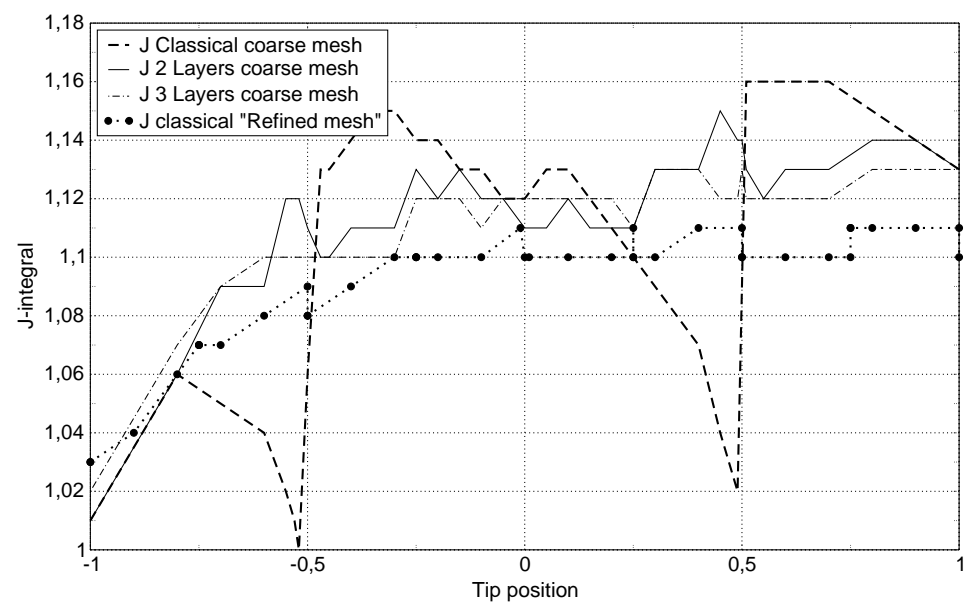

Figure 10: Evolution of the tearing energy with respect to the crack tip position.

energy of the sample without a crack by :

$$
k=-\frac{\Delta U}{W H c^{2}}
$$

where $W$ stands for the uniform strain energy density in the domain before the introduction of the crack, $c$ is the half length of the crack, $H$ is the thickness of the plate in its reference configuration, and $\Delta U$ represents the loss of energy which stems from the crack. Introducing the $J$-integral, it reduces to :

$$
k=\frac{J}{2 W c}
$$

Finally, Rivlin \& Thomas also argued that this parameter should only depend on the stretch level. 


\subsubsection{Comparison with Greensmith experiments}

Greensmith performed experiments on SET samples [31] held in simple extension. His results were used by Lindley in addition to finite element simulations to propose an empirical relation between $k$ and the stretch level in the tensile direction $\lambda$ [32] :

$$
k=\frac{2.95-0.008(1-\lambda)}{\sqrt{\lambda}}
$$

The reference mesh (12958 dofs) presented in Figure 11(a) is used to establish a reference

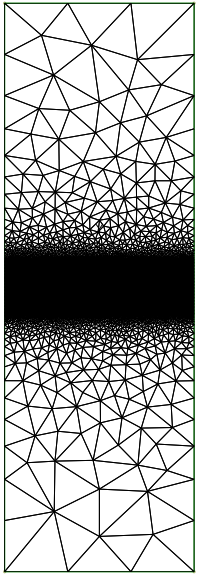

(a)

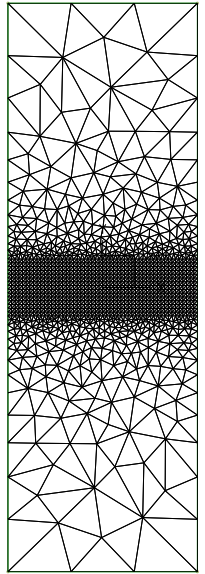

(b)

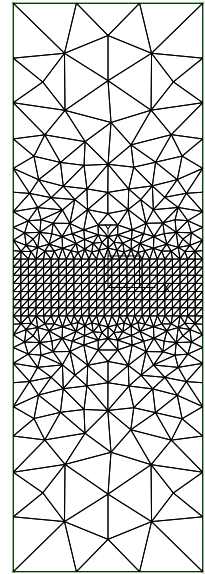

(c)

Figure 11: (a) Reference mesh (12958 dofs); (b) Fine mesh (2664 dofs) ; (c) Coarse mesh (912 dofs).

solution. Evolution of the $J$-integral $J_{\mathrm{NL}}$ and of its geometrically linear counterpart $J_{\mathrm{LIN}}$ (assuming small displacements and strains i.e. replacing $\underline{\underline{P}}$ by the Cauchy stress tensor, and performing derivations with respect to the current configuration) are computed with respect to the stretching level. The corresponding results are presented in Figure 12. The difference between $J_{\mathrm{NL}}$ and $J_{\mathrm{LIN}}$ curves shows the influence of the "blunting" of the crack tip with large displacements; this leads to a lower value for the nonlinear expression of the tearing energy. However, linear and nonlinear curves match at small strains. We also remark that $J_{\mathrm{NL}}$ is close to $J_{\text {Lindley }}$, which shows the reliability of the method. We study also the influence of the enrichment by considering a fine (2664 dofs) and a coarse (912 dofs) mesh presented respectively in Figure 11(b) and (c). The errors on the $J$ integrals between the results obtained with those meshes and the reference solution are shown in Figure 13 as a function of the stretch level, with or without near tip enrichment. The two curves ("Fine mesh (1664 dofs) - H(X) \& $r^{1 / 2} \sin (\theta / 2)$ " and "Fine mesh (2664 dofs) - $H(X))$ describe the influence of the enrichment on the fine mesh, and the two other ( "Coarse mesh (912 dofs) H(X) and $r^{1 / 2} \sin (\theta / 2)$ " and "Coarse mesh (912 dofs) $H(X))$ exhibits its influence on the coarse mesh. As seen, this influence is much more 


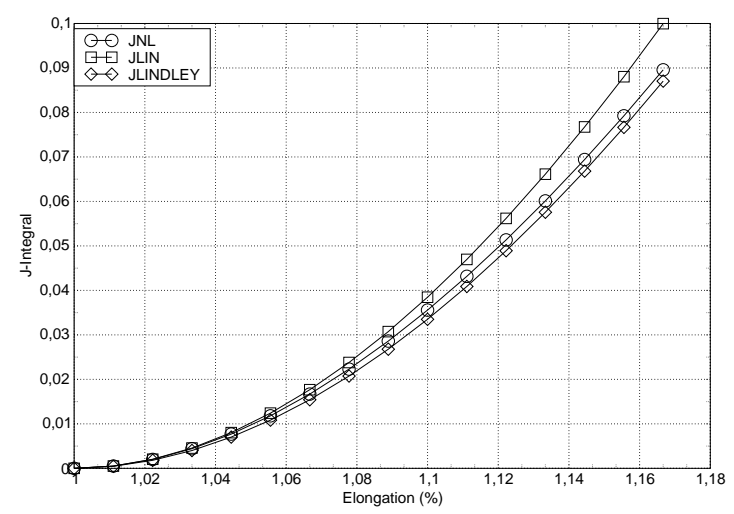

Figure 12: Evolution of the tearing energy with respect to the stretch level.

significant when dealing with coarse meshes (decrease of $2.7 \%$ on the error for the coarse mesh and $0.6 \%$ for the fine one).

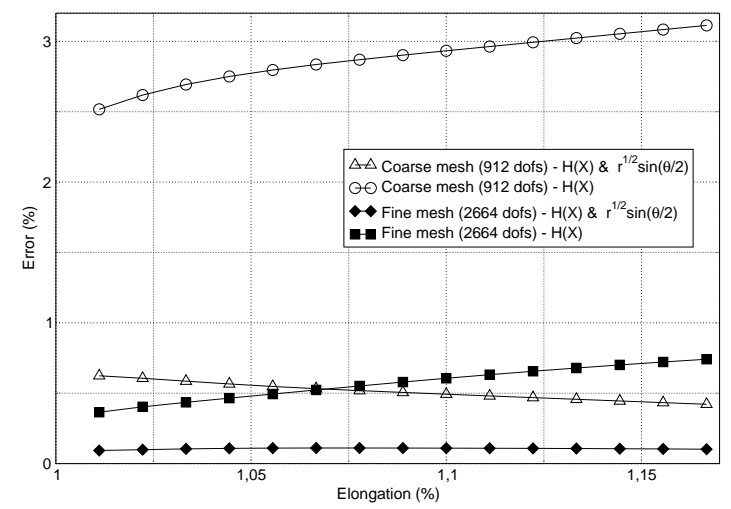

Figure 13: Influence of the mesh and the enrichment on the tearing energy with respect to the stretch level.

\subsubsection{Griffith problem}

Consider a Griffith cracked membrane in nonlinear elasticity as shown in Figure 14(a). The "infinite" membrane has a center crack of length equal to $0.5 \mathrm{~mm}$. It is subjected to three fundamental loadings as depicted in Figure 14: (a) uniaxial extension, (b) equibiaxial extension and (c) pure shear [33]. Dimensions of the domain are chosen so that the crack can be considered in detail, i.e. $h=w=6 \mathrm{~mm}$ for uniaxial and equibiaxial extension and $h=6 \mathrm{~mm}$ and $w=60 \mathrm{~mm}$ for pure shear. For the two first problems, a $1 \mathrm{MPa}$ tensile loading is prescribed and rigid body motions are blocked. For pure shear, a $6 \mathrm{~mm}$ vertical displacement is prescribed on the bottom edge of the domain. Problem 
symmetries are not taken into account in the model in order to check if the $J$-integral results are similar for both tips of the crack. Three different meshes are considered: a very fine mesh (19758 dofs) to compute the reference solution (see Figure 15), a fine mesh (7234 dofs), and a coarse mesh (2176 dofs) depicted in Figures 16 and 17 respectively. The domain used to compute the tearing energy is a $0.2 \mathrm{~mm}$ radius circle in all cases. First, it is to mention that the difference of $J$-integral values obtained at the two tips is below $0.02 \%$ in all cases. The evolution of the tearing energy versus the stretch

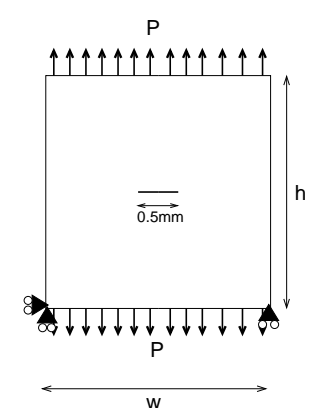

(a)

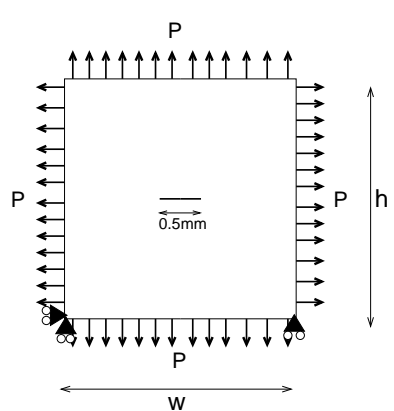

(b)

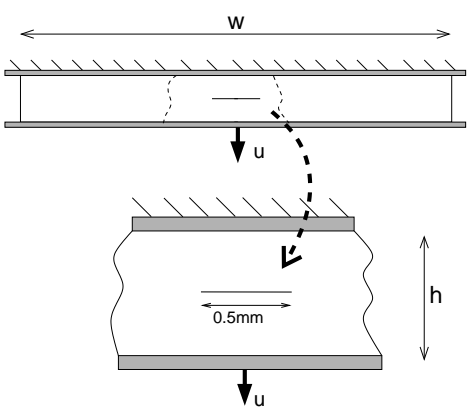

(c)

Figure 14: Nonlinear Griffith problem: (a) uniaxial extension, (b) equibiaxial extension, (c) pure shear.

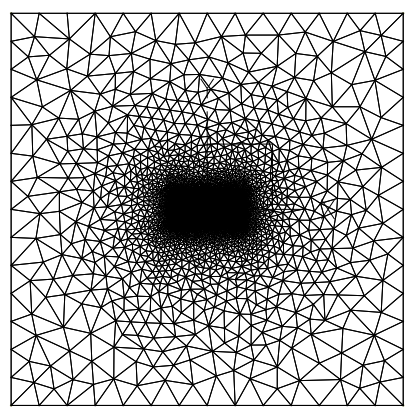

(a)

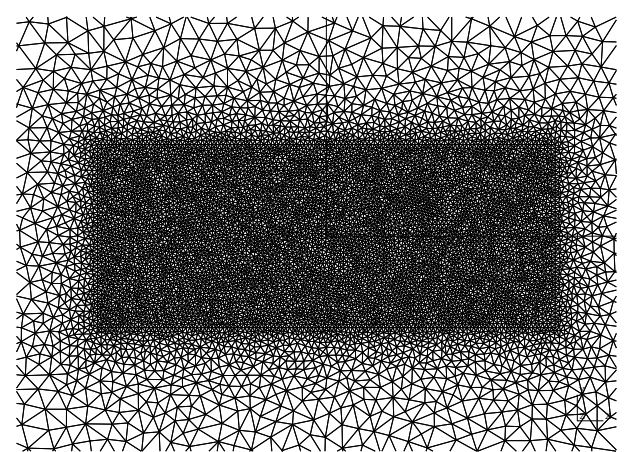

(b)

Figure 15: (a) The "reference" FE mesh (19758 dofs), (b) Zoom at the crack area.

level is plotted for uniaxial tension in Figure 18. The results are compared with the approximation of $k$ proposed by Lake for central cracks held in simple tension [34]. The factor is approximated by equating the energy loss due to crack opening to the energy required to close it again :

$$
k=\frac{\pi}{\sqrt{\lambda}}
$$

Note that this expression was obtained considering a geometrically nonlinear approach, 


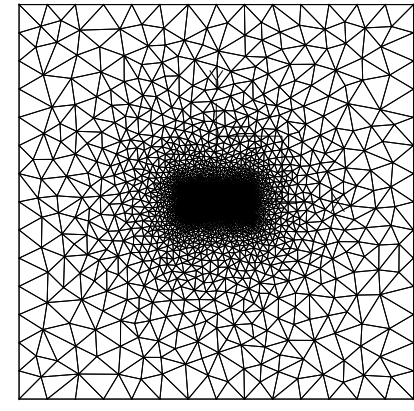

(a)

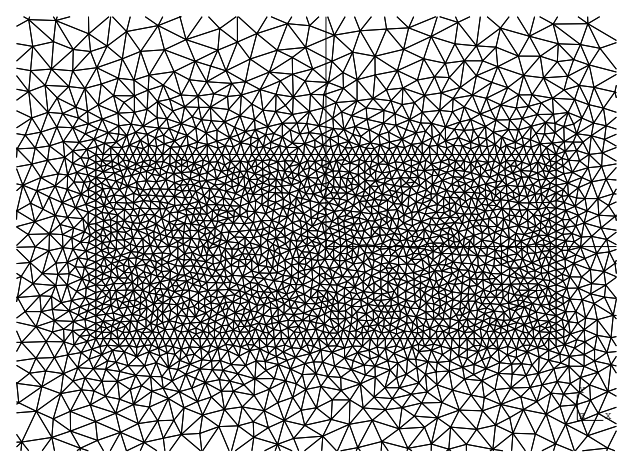

(b)

Figure 16: (a) The "fine" FE mesh $\left(\mathrm{N}^{\circ} 1\right)$ (7234 dofs), (b) Zoom at the crack area.

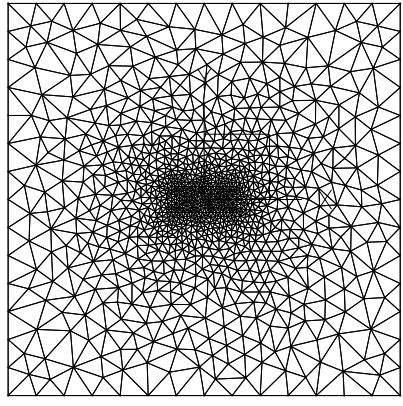

(a)

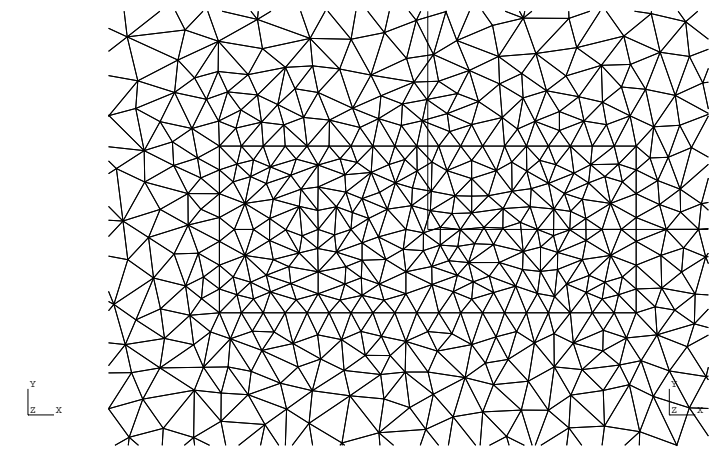

(b)

Figure 17: (a) The "coarse" FE mesh ( $\left.{ }^{\circ} 2\right)$ (2176 dofs), (b) Zoom at the crack area.

but a linear constitutive equation. As a consequence, Eq. (30) is not accurate for large strains (greater than 100\%). Lindley's approximation of $J$ has also been plotted (as in ref. [35]) even if both curves cannot be compared (the SET boundary conditions are different from those of a symmetric Griffith problem). As in the previous Section, the influence of the crack tip blunting is noticeable and all the curves overlap at for small strains.

The influence of the enrichment is studied by considering the evolution of the $J$-integral as a function of stretch level for the two meshes and for various enrichment functions. More precisely, in the enrichment functions in Eq. (22), the singularity degree of $u_{2}$ is changed from $1 / 2$ to $1 / 4,1 / 3,1$ and 2 . The discrepancy between the corresponding results and the reference solution is shown in Figure 19. The singularity degree strongly influences the accuracy of the solution: as the singularity degree tends to its theoretical value, i.e. $1 / 2$ the results approach the reference solution. The best enrichment functions are clearly those obtained by considering analytical results. Moreover, the case without 


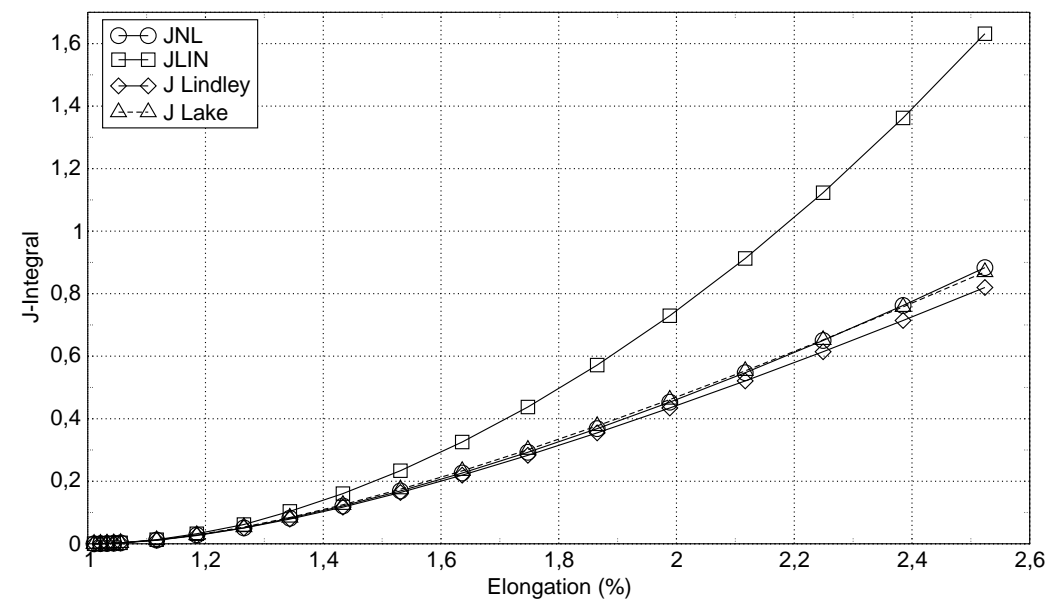

Figure 18: Evolution of $J_{\mathrm{LIN}}$ and $J_{\mathrm{NL}}$ (uniaxial extension).

enrichment is also considered in Figure 19: the results show that for large strains (due to blunting), it is better to not enrich the crack tip than make a poor choice of enrichment.

In order to examine the validity of our developments under different loading conditions, equibiaxial extension and simple shear loading cases presented above in Fig. 14(b) and (c) are considered. For equibiaxial extension, the coarse mesh $\left(\mathrm{n}^{\circ} 2\right)$ is used and a 4372 dofs mesh is considered for the pure shear case. The evolution of $k$ as a function of stretch level is presented in Figure 20(a) and (b) for equibiaxial and pure shear respectively. These results are compared with those obtained by Yeoh [35], who approximates $k$ using a method based on the crack surface displacement [36], assuming that the deformed crack admits an elliptical shape. The numerical results are in good agreement, even if the X-FEM values are slightly different because of the enrichment. Moreover, the calculated small strain limit of $k$ agrees well with theoretical values as shown in Table 1.

\begin{tabular}{|c|c|c|}
\hline Loading conditions & Theoretical values & X-FEM values \\
\hline Uniaxial extension & $k=\pi$ & $k=3.17$ \\
Equibiaxial extension & $k=\pi$ & $k=3.16$ \\
Pure shear & $k=\frac{4}{3} \pi \simeq 4.18$ & $k=4.08$ \\
\hline
\end{tabular}

Table 1: Small strain value of $k$.

\subsubsection{Membrane with an inclined center crack}

As a last example, we consider an "infinite membrane" with an inclined center crack subjected to uniaxial tension. The membrane, presented in Figure 21, is loaded on its 


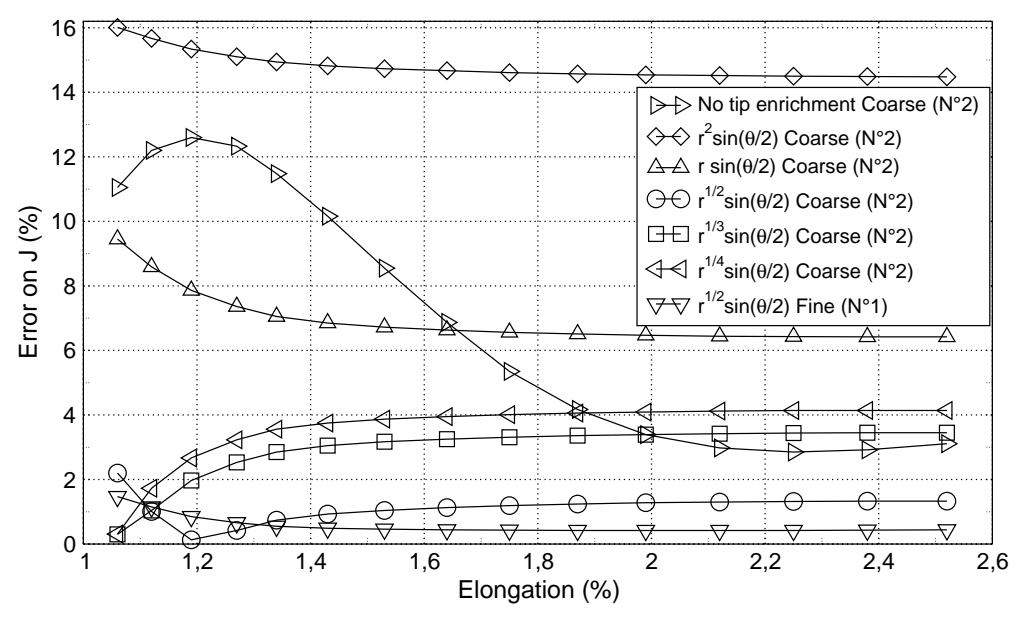

Figure 19: Influence of the enrichment for the two meshes: error on the value of the energy release rate (\%).

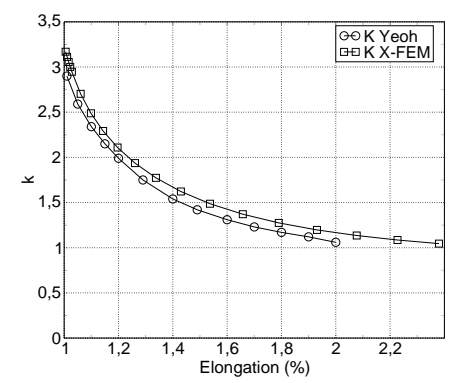

(a) Equibiaxial extension

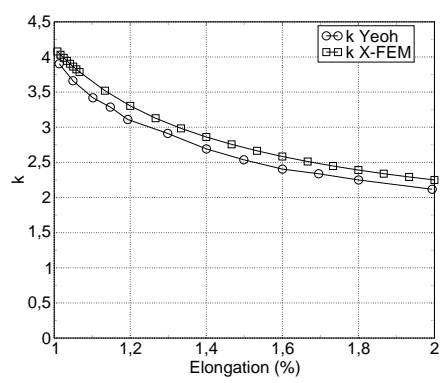

(b) Pure shear

Figure 20: Evolution of $k$ with respect of the elongation.

top and bottom faces. The corresponding Piola-Kirchhoff $(P)$ traction evolves from 0 to $0.7 \mathrm{MPa}$. A crack of length $2 a$ parametrized by its inclination angle $\beta\left(\beta \in\left[0^{\circ} ; 90^{\circ}\right]\right)$ is located at the center of the membrane. The membrane is considered as a square $(h=w)$ whose dimensions are far greater than those of the crack $(2 a / w<0.1)$. A 12734 dofs fine mesh is considered. The evolution of the tearing energy non-dimensionalized with respect to its value for $\beta=0$ is plotted as a function of $\beta$ for various stretch levels in Figure 22(a). In the present case, this evolution does not depend on the stretch level. Moreover, this result is close to the linear elastic fracture mechanics solution for which the tearing energy is related to $\beta$ by :

$$
J=\frac{\sigma^{2} \pi}{E}\left[a\left(\cos ^{4}(\beta)+\sin (\beta)^{2} \cos (\beta)^{2}\right)\right]
$$




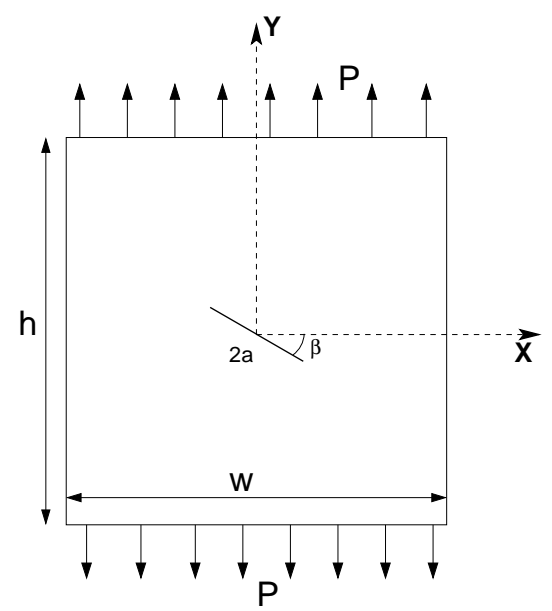

Figure 21: Plate with a center crack at angle $\beta$.

where $\sigma$ is the applied traction on the top edge, $E$ is the Young Modulus, and $\beta$ is the crack inclination angle. $J$ can be written as :

$$
J(\sigma, a, \beta)=J_{\sigma}(\sigma) \cdot J_{C}(a, \beta)
$$

The difference between the large strain solutions of Fig. 22(a), and the linear solution

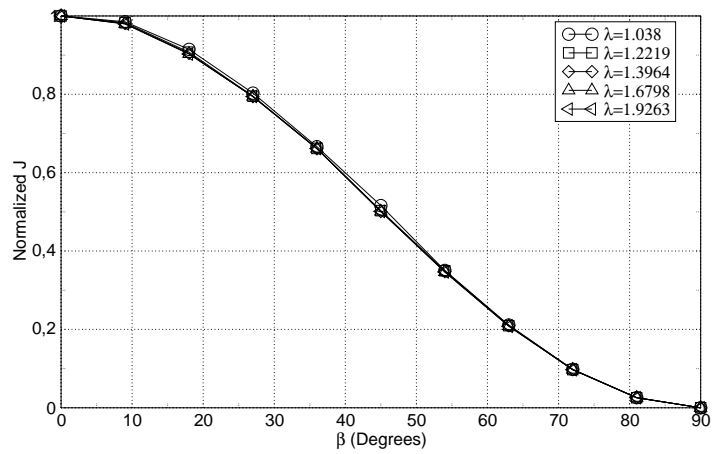

(a)

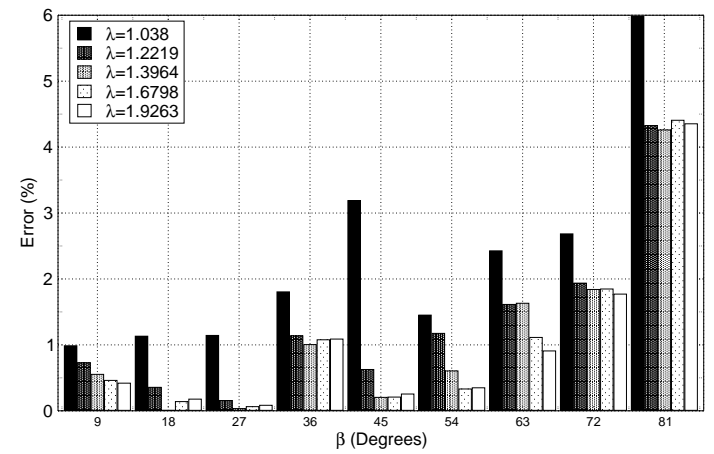

(b)

Figure 22: (a) Evolution of normalized J, (b) Evolution of the error on (31).

Eq. (31) is presented in Figure 22(b). It remains below 6\%. According to these results, we investigate the possibility of establishing a nonlinear counterpart to Eq. (31). In regards to Fig. 22(b) the dependence of $J_{\mathrm{NL}}$ on $\beta$ is identical to the dependence of $J$ on $\beta$ in Eq. (31). Thus only the dependence of $J_{\mathrm{NL}}$ on $P$ and $a$ should be studied. The corresponding results are given in Figure 23(a) and (b) for the dependence on $P$ and $a$ respectively. Examining these figures, it is possible to separate the nonlinear tearing energy into the product of a function of the stress state only and a function of the crack 
geometry only:

$$
\begin{aligned}
J_{\mathrm{NL}}(P, a, \beta) & =J_{N L-P}(P) \times J_{N L-C}(a, \beta) \\
& =b P^{2.1} J_{N L-C}(a, \beta)
\end{aligned}
$$

where $b$ is a factor that depends on the material behaviour. As a final remark, it should be noted that the previous results may not be valid for other constitutive equations and for plane strain or 3D problems, and that other geometries have not been checked.

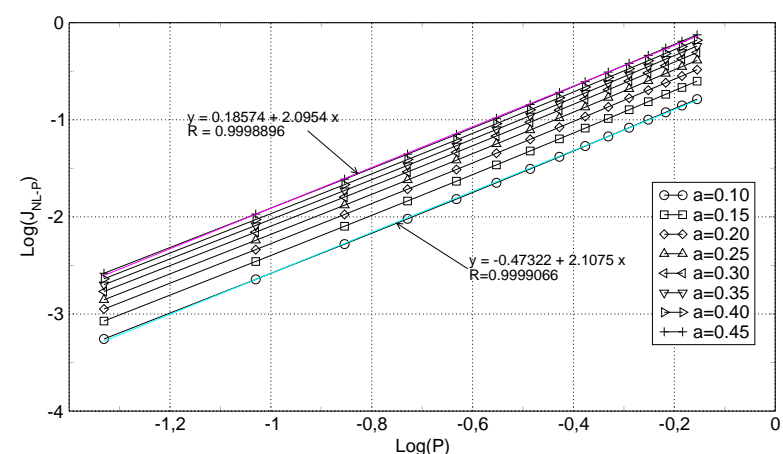

(a)

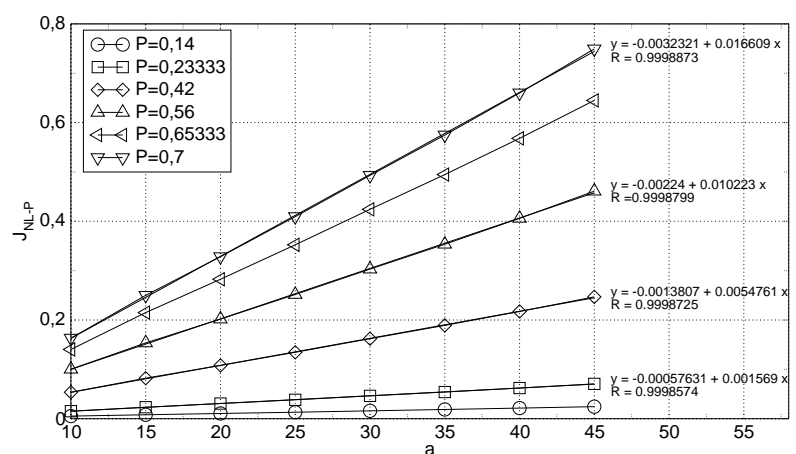

(b)

Figure 23: (a) $\log \left(J_{N L-P}\right)$ vs. $\log (\mathrm{P})$, (b) $J_{N L-P}$ vs. crack length.

\section{CONCLUSION}

This work has presented an improvement of the eXtended Finite Element Method for large strain hyperelastic fracture mechanics. The asymptotic displacement field near the tip of a crack in a Neo-Hookean material has been discussed to obtain suitable enrichment functions for plane stress problems. Numerical examples showed the reliability of the approach and the influence of enrichment functions on results obtained with coarse meshes. Validations also demonstrated that interesting results could be easily obtained with this method. The present study is therefore a first step to overcome the lack of simulation tools for rubber fracture mechanics pointed out in [1].

However, some particular points still have to be investigated. First, the enrichment function considered here is only valid for the Neo-Hookean material constitutive equation and for plane stress problems. Proper enrichment functions should be investigated in order to consider more realistic material models, such as Mooney-Rivlin or Ogden constitutive equations. Another point to investigate is the enforcement of the incompressibility constraint for 3D problems. A first work has already been proposed by Dolbow and Devan [14] for plane strain problems, adapting the enhanced assumed strain method to the eXtended Finite Element Method .

\section{ACKNOWLEDGMENTS :}

The authors greatfully acknowledge Prof. J. Dolbow for useful discussions. 


\section{References}

[1] P.Charrier E.O.Kuczynski E.Verron G.Marckmann L.Gornet and G.Chagnon. Theorical and numerical limitations for the simulation of crack propagation in natural rubber components. In Constitutive Models for Rubber III, Busfield \& Muhr (eds), 2003.

[2] M. Fleming, Y.A. Chu, B. Moran, and T. Belytschko. Enriched element-free galerkin methods for crack tip fields. International Journal for Numerical Methods in Engineering, 1997.

[3] I. Babuska and J. Y.Melenk. The partition of unity finite element method. International Journal for Numerical Methods in Engineeringe, 40(4):727-758, February 1997.

[4] T. Belytschko and T. Black. Elastic crack growth in finite elements with minimal remeshing. International Journal for Numerical Methods in Engineering, 45(5):601$620,1999$.

[5] N. Moës, J. Dolbow, and T. Belytschko. A finite element method for crack growth without remeshing. International Journal for Numerical Methods in Engineering, 46:131-150, 1999 .

[6] J. Dolbow, N. Moës, and T. Belytschko. An extended finite element method for modeling crack growth with frictional contact. Comp. Meth. in Applied Mech. and Engrg., 190:6825-6846, 2001.

[7] C. Daux, N. Moës, J. Dolbow, N. Sukumar, and T. Belytschko. Arbitrary branched and intersecting cracks with the eXtended Finite Element Method. International Journal for Numerical Methods in Engineering, 48:1741-1760, 2000.

[8] N. Moës, A. Gravouil, and T. Belytschko. Non-planar 3D crack growth by the extended finite element and level sets. part I: Mechanical model. International Journal for Numerical Methods in Engineering, 53:2549-2568, 2002.

[9] A. Gravouil, N. Moës, and T. Belytschko. Non-planar 3d crack growth by the extended finite element and level sets. part II: level set update. International Journal for Numerical Methods in Engineering, 53:2569-2586, 2002.

[10] G.N. Wells and L.J. Sluys. A new method for modelling cohesive cracks using finite elements. International Journal for Numerical Methods in Engineering, 50:2667$2682,2001$.

[11] N. Moës and T. Belytschko. Extended finite element method for cohesive crack growth. Engineering Fracture Mechanics, 69:813-834, 2002.

[12] J.A. Sethian. Fast marching methods and level sets methods for propagating interfaces. 
[13] M. Stolarska, D.L. Chopp, N. Moës, and T. Belytschko. Modelling crack growth with level-set and the extended finite element method. International journal for numerical methods in Engineering, 51:943-960, 2001.

[14] J.E. Dolbow and A. Devan. Enrichment of enhanced assumed strain approximations for representing strong discontinuities : Addressing volumetric incompressibility and the discontinuous patch test. International journal for numerical methods in engineering, 59:47-67, 2004.

[15] A.A. Griffith. The phenomena of rupture and flow in solids. Transactions of Royal Society London, 221, 1920.

[16] R.S. Rivlin et A.G. Thomas. Rupture of rubber. i. characteristic energy for tearing. Journal for Polymer science, 3:291-318, 1953.

[17] P. Steinmann. Application of material forces to hyperelastostatic fracture mechanics. i. continuum mechanical setting. International Journal of Solids and Structures, 37:7371-7391, 2000 .

[18] Ph. Destuynder, M. Djaoua, and S. Lescure. Quelques remarques sur la mecanique de la rupture elastique. Jounrla de mecnique theorique et appliquee, 2(1):113-135, 1983.

[19] B. Moran and C.F. Shih. Crack tip and associated domain integrals from momentum and energy balance. Engineering Fracture Mechanics, 127:615-642, 1987.

[20] M. Stolarska, D. L. Chopp, N. Moës, and T. Belytschko. Modelling crack growth by level sets and the extended finite element method. International Journal for Numerical Methods in Engineering, 51(8):943-960, 2001.

[21] J. Dolbow, N. Moës, and T. Belytschko. Modeling fracture in Mindlin-Reissner plates with the eXtended finite element method. Int. J. Solids Structures, 37:7161$7183,2000$.

[22] Z. Goangseup and T. Belytschko. New crack-tip elements for x-fem and applications to cohesive cracks. international journal for numerical methods in engineering, $57: 2221-2240,2003$.

[23] P.H. Geubelle and W.G. Knauss. Finite strains at the tip oh a crack in a sheet of hyperelastic material : I. homogeneous case. Journal of Elasticity, 35:61-98, 1994.

[24] P.H. Geubelle and W.G. Knauss. Finite strains at the tip oh a crack in a sheet of hyperelastic material : Ii. special bimaterial case. Journal of Elasticity, 35:99-137, 1994.

[25] P.H. Geubelle and W.G. Knauss. Finite strains at the tip oh a crack in a sheet of hyperelastic material : Iii. general bimaterial case. Journal of Elasticity, 35:139-174, 1994. 
[26] J.K. Knowles and E. Sternberg. An asymptotic finite-deformation analysis of the elastostatic field near the tip of a crack. Journal of Elasticity, 3:67-107, 1973.

[27] J.K. Knowles and E. Sternberg. Finite-deformation analysis of the elastostatic field near the tip of a crack : Reconsideration of higher order results. Journal of Elasticity, 4:201-233, 1974.

[28] R.A. Stephenson. The equilibrium field near the tip of a crack for finite plane strain of incompressible elastic matreials. Journal of Elasticity, 12:65-99, 1982.

[29] HKS. Abaqus/Standard user manual.

[30] J. Rice. A path independent integral and the approximation analysis of strain concentration by notches and cracks. Journal of Applied Mechanics, 35:379-386, 1968.

[31] H.W. Greensmith. Rupture of rubber part 10 : The changes in stored energy on making a small cut in a test piece held in simple extension. J. Appl. Polym. Sci., 7:993-1002, 1963.

[32] P.B. Lindley. Energy for crack growth in model rubber components. J. Strain Anal., 7:132-140, 1972.

[33] L. R. G. Treloar. Stress-strain data for vulcanised rubber under various types of deformation. Trans. Faraday Soc., 40:59-70, 1944.

[34] G.J. Lake. Application of fracture mechanics to failure in rubber articles, with particulary reference to groove cracking in tyres. Int. Conf. Yeld, Deformation and fracture of polymers, Cambridge, 1970.

[35] O.H. Yeoh. Relation between crack surface displacement and strain energy release rate in thin rubber sheets. Mechanics of Materials, 32:459-474, 2002.

[36] P.L. Key. A relation between crack surface displacement and the strain energy release rate. Int. J. Fracture Mech., 5:287-296, 1969. 